August 2006

DAMTP-06-62

UT-06-16

hep-th/0608047

\title{
On the Scattering of Magnon Boundstates
}

\author{
Heng-Yu Chen ${ }^{1}$, Nick Dorey ${ }^{1}$ and Keisuke Okamura ${ }^{2}$ \\ ${ }^{1}$ DAMTP, Centre for Mathematical Sciences \\ University of Cambridge, Wilberforce Road \\ Cambridge CB3 0WA, UK \\ and \\ ${ }^{2}$ Department of Physics, Faculty of Science, \\ University of Tokyo, Bunkyo-ku, \\ Tokyo 113-0033, Japan.
}

\begin{abstract}
We study the scattering of magnon boundstates in the spin-chain description of planar $\mathcal{N}=4$ SUSY Yang-Mills. Starting from the conjectured exact S-matrix for magnons in the $S U(2)$ sector, we calculate the corresponding S-matrix for boundstates with an arbitrary number of constituent magnons. The resulting expression has an interesting analytic structure with both simple and double poles. We also calculate the semiclassical S-matrix for the scattering of the corresponding excitations on the string worldsheet known as Dyonic Giant Magnons. We find precise agreement with the magnon boundstate S-matrix in the limit of large 't Hooft coupling.
\end{abstract}


The discovery of integrability in planar $\mathcal{N}=4$ SUSY Yang-Mills [1, 2] and in string theory on $A d S_{5} \times S^{5}$ [3] has lead to considerable progress in testing AdS/CFT duality. In particular, an increasingly precise correspondence between the spin-chain description of gauge theory anomalous dimensions and the world-sheet theory of the dual string continues to emerge 4, 5, 6, 7, 8, 9]. The correspondence is clearest in a limit where a $U(1)$ R-charges $J_{1}$ and scaling dimensions $\Delta$ of gauge theory operators become large. Following $[9]^{1}$, the specific limit we consider is one where $J_{1}$, $\Delta \rightarrow \infty$ with the difference $E=\Delta-J_{1}$ and the 't Hooft coupling $\lambda$ held fixed. In this limit the spin chain/string becomes infinitely long and the spectrum consists of local excitations which propagate freely apart from pairwise scattering. The physical content of the limiting theory is the spectrum of asymptotic states and their S-matrix and the main problem is to compare the spectrum and S-matrix which appear on both sides of the correspondence.

The asymptotic spectrum of the gauge theory spin chain includes an infinite tower of BPS states labelled by a positive integer $Q$, which also carry a conserved momentum $p$. The state $Q=1$ corresponds to the fundamental spin-chain excitation known as a magnon. States with $Q>1$ correspond to boundstates of $Q$ fundamental magnons [13. Each of these states lives in a short representation of supersymmetry and has an exact dispersion relation,

$$
E=\sqrt{Q^{2}+8 g^{2} \sin ^{2}\left(\frac{p}{2}\right)}
$$

where, following the convention of [4], we introduce a coupling $g$ which is related to the 't Hooft coupling $\lambda$ by $g^{2}=\lambda / 8 \pi^{2}$. For $Q>1$, this formula is a generalisation of the exact [8] magnon dispersion relation obtained in [4, 6, 7] (see also [14).

In the following we will study the scattering of the BPS states described above. The exact S-matrix for the magnons themselves is known up to a single overall phase [8]. In the $S U(2)$ sector, the remaining ambiguity corresponds to the dressing factor first introduced in [5, 15. As we review below (see Eqn (12)), the dressing factor takes a very specific form as a function of the conserved charges of the theory but still involves an infinite number of undetermined coefficients. In an integrable theory, the scattering of boundstates is uniquely determined by the scattering of their constituents [16, 17, 18]. In this Letter, we will take the exact magnon S-matrix, including the dressing factor, as a starting point and derive the corresponding S-matrix for the scattering of magnon boundstates in the $S U(2)$ sector. The resulting S-matrix has an interesting analytic structure with simple poles corresponding to boundstate contributions in the s- and t- channels as well as double-poles corresponding to anomalous thresholds. The boundstate S-matrix also

\footnotetext{
${ }^{1}$ For related earlier work see [10, 11, 12].
} 
includes a dressing factor which is functionally identical to the one appearing in the fundamental magnon S-matrix. We note that this universality of the dressing factor is essentially equivalent to its conjectured form as a function of the conserved charges mentioned above.

On the string theory side, the fundamental magnons and their boundstates correspond to solitons of the worldsheet theory which can be studied using semiclassical methods for $g>>1$ ]. In particular, $S U(2)$ sector boundstates with values of $Q$ which scale linearly with $g$, are identified with classical string configurations known as "Dyonic Giant Magnons" [19, 20, 21, 22, 23, 24]. In string theory, $Q$ corresponds to a conserved angular momentum on $S^{5}$ and the exact dispersion relation (10) is already obeyed at the classical level. These string configurations can also be mapped to soliton solutions of the Complex sine-Gordon ( $\mathrm{CsG}$ ) equation using a certain reduction of the worldsheet $\sigma$-model [19]. In the following we will use the CsG description to obtain a semiclassical approximation to the S-matrix for the Dyonic Giant Magnons. Our main result is that this precisely matches the large- $g$ limit of the magnon boundstate S-matrix described above. A similar comparison in the $Q=1$ case of fundamental magnons was performed in [9]. A new feature of the present case is that both the dressing factor and the remaining factor which originates from the all-loop gauge theory Bethe ansatz of [4] contribute at leading order in the $g \rightarrow \infty$ limit and therefore both parts are tested by the comparison. While this work was being completed we learned of a forthcoming paper [33] with similar results.

A generic asymptotic state in the $S U(2)$ sector has two independent quantum numbers $p$ and $Q$. It will be convenient to use an alternative parametrisation in terms of two complex variables $X^{ \pm}$ with,

$$
\exp (i p)=\frac{X^{+}}{X^{-}}
$$

The energy and charge are given respectively as,

$$
\begin{aligned}
& E=\frac{g}{i \sqrt{2}}\left[\left(X^{+}-\frac{1}{X^{+}}\right)-\left(X^{-}-\frac{1}{X^{-}}\right)\right], \\
& Q=\frac{g}{i \sqrt{2}}\left[\left(X^{+}+\frac{1}{X^{+}}\right)-\left(X^{-}+\frac{1}{X^{-}}\right)\right] .
\end{aligned}
$$

If $Q$ and $E$ are regarded as free complex parameters then $X^{+}$and $X^{-}$are unconstrained complex variables. The condition of fixed integer charge $Q$ provides a cubic constraint on $X^{ \pm}$which defines a complex torus [11. The case $Q=1$ corresponds to the fundamental magnon and the variables $X^{ \pm}$coincide with the usual spectral parameters $x^{ \pm}$. We will reserve the use of lower-case variables $x^{ \pm}, y^{ \pm}, \ldots$ for this special case. For any positive integer $Q$, physical states with real momentum and positive energy are obtained by imposing the conditions $X^{-}=\left(X^{+}\right)^{*}$ and $\left|X^{ \pm}\right|>1$. 
It will be useful to define a rapidity variable associated with each $\operatorname{state}^{2}$,

$$
U(X) \equiv U\left(X^{+}, X^{-}\right)=\frac{1}{2}\left[\left(X^{+}+\frac{1}{X^{+}}\right)+\left(X^{-}+\frac{1}{X^{-}}\right)\right] .
$$

Following [25] we also define a basis for the higher conserved charges carried by the BPS states,

$$
q_{r}(X) \equiv q_{r}\left(X^{+}, X^{-}\right)=\frac{i}{r-1}\left[\frac{1}{\left(X^{+}\right)^{r-1}}-\frac{1}{\left(X^{-}\right)^{r-1}}\right], \quad r=2,3, \ldots
$$

The rapidity and higher conserved charges, can also be given explicitly in terms of the momentum $p$ and charge $Q$, (see also [21, 26])

$$
\begin{aligned}
& U(p, Q)=\frac{1}{g \sqrt{2} \cot \left(\frac{p}{2}\right) \sqrt{Q^{2}+8 g^{2} \sin ^{2}\left(\frac{p}{2}\right)}}, \\
& q_{r}(p, Q)=\frac{2 \sin \left(\frac{r-1}{2} p\right)}{r-1}\left(\frac{\sqrt{Q^{2}+8 g^{2} \sin ^{2}\left(\frac{p}{2}\right)}-Q}{2 \sqrt{2} g \sin \left(\frac{p}{2}\right)}\right)^{r-1}, \quad r=2,3, \ldots .
\end{aligned}
$$

As a consequence of integrability, the asymptotic states described above undergo factorised scattering. In other words the S-matrix for the scattering of an arbitrary number of excitations can be written consistently as a product of two-body factors. In the case of fundamental magnons in the $\mathrm{SU}(2)$ sector, with spectral parameters $x^{ \pm}$and $y^{ \pm}$respectively, the exact two body S-matrix can be written as,

$$
s(x, y) \equiv s\left(x^{+}, x^{-} ; y^{+}, y^{-}\right)=\hat{s}(x, y) \times \sigma(x, y)
$$

The first factor,

$$
\hat{s}(x, y)=\frac{x^{+}-y^{-}}{x^{-}-y^{+}} \frac{1-1 / x^{+} y^{-}}{1-1 / x^{-} y^{+}}=\frac{U(x)-U(y)+\frac{\sqrt{2} i}{g}}{U(x)-U(y)-\frac{\sqrt{2} i}{g}}
$$

originates in the all-loop asymptotic Bethe ansatz of Beisert, Dippel and Staudacher (BDS) 44. In the following we will refer to it as the BDS factor. This factor has a pole in the physical region of the spectral plane at the point $x^{-}=y^{+}$which corresponds to the formation of the $Q=2 \operatorname{BPS}$ boundstate in the s-channel [13]. The second term, known as the "dressing factor" [5] corresponds to the most general long-range integrable deformation of the Heisenberg spin chain [15]. It can be written as,

$$
\sigma(x, y)=\exp (i \theta(x, y))
$$

where,

$$
\theta(x, y)=\sqrt{2} g \sum_{r=2}^{\infty} \sum_{n=0}^{\infty} c_{r, r+1+2 n}(g)\left[q_{r}(x) q_{r+1+2 n}(y)-q_{r}(y) q_{r+1+2 n}(x)\right] .
$$

\footnotetext{
${ }^{2}$ Here and in the following we will use the shorthand notation $f(X)$ to denote $f\left(X^{+}, X^{-}\right)$defined as a function of two independent variables.
} 
Here $q_{r}$ are the higher charges defined in (6) and an infinite tower of unknown coefficients $c_{r, s}$ depending on the coupling constant $g$ remain to be determined. Agreement with classical string theory uniquely determines the large- $g$ behaviour of these coefficients as [5],

$$
c_{r, s}(g)=\delta_{r+1, s}+\mathcal{O}\left(\frac{1}{g}\right) .
$$

More recently, the leading corrections in $1 / g$ have been determined by comparison with the one-loop corrections in the string $\sigma$-model [27].

An important feature of the general form (12) is that it is bilinear in the conserved charges $q_{r}$. As a consequence, the scattering phase $\theta(x, y)=\theta\left(x^{+}, x^{-} ; y^{+}, y^{-}\right)$is seperately odd under the interchange of $x^{+}$and $x^{-}$and under the interchange of $y^{+}$and $y^{-}$. Because of unitarity, $\theta$ is also odd under the interchange $x^{ \pm} \leftrightarrow y^{ \pm}$. In fact, the general form (12), together with (6), implies that we can write $\theta$ in terms of a function $k(x, y)$ of two variables satisfying $k(x, y)=-k(y, x)$ as,

$$
\theta\left(x^{+}, x^{-}, y^{+}, y^{-}\right)=\sqrt{2} g\left\{k\left(x^{+}, y^{+}\right)+k\left(x^{-}, y^{-}\right)-k\left(x^{+}, y^{-}\right)-k\left(x^{-}, y^{+}\right)\right\}
$$

As above $\theta$ must scale like $g$ in the strong coupling limit. Thus the function $k$ has an expansion of the form,

$$
k(x, y)=k_{0}(x, y)+\frac{1}{g} k_{1}(x, y)+\mathcal{O}\left(\frac{1}{g^{2}}\right)
$$

and the leading term can be deduced from the leading order result (13) to be [12],

$$
k_{0}(x, y)=-\left[\left(x+\frac{1}{x}\right)-\left(y+\frac{1}{y}\right)\right] \log \left(1-\frac{1}{x y}\right)
$$

Let us now consider two magnon boundstates with charges $Q_{1} \geq Q_{2}$ and momenta $p_{1}$ and $p_{2}$ respectively. Equivalently we can describe these states with spectral parameters $X^{+}, X^{-}, Y^{+}$and $Y^{-}$with,

$$
\exp \left(i p_{1}\right)=\frac{X^{+}}{X^{-}}, \quad \exp \left(i p_{2}\right)=\frac{Y^{+}}{Y^{-}}
$$

where $X^{ \pm}$satisfies (4) with $Q=Q_{1}$ and a similar equation holds for $Y^{ \pm}$with $Q=Q_{2}$. Our goal is to find the S-matrix $S(X, Y)$ describing the scattering of these two boundstates states. In an integrable quantum theory, the S-matrix for the scattering of boundstates is uniquely determined by the S-matrix of their constituents. Thus, in the present case, we begin by considering the scattering of $Q_{1}+Q_{2}$ fundamental magnons with individual spectral parameters,

$$
x_{j_{1}}^{ \pm}, \quad y_{j_{2}}^{ \pm} \quad \text { with } j_{1}=1, \ldots, Q_{1}, j_{2}=1, \ldots, Q_{2} .
$$


As above the spectral parameters for fundamental magnons satisfy the constraints,

$$
\begin{aligned}
& \left(x_{j_{1}}^{+}+\frac{1}{x_{j_{1}}^{+}}\right)-\left(x_{j_{1}}^{-}+\frac{1}{x_{j_{1}}^{-}}\right)=i \frac{\sqrt{2}}{g}, \quad j_{1}=1, \ldots, Q_{1}, \\
& \left(y_{j_{2}}^{+}+\frac{1}{y_{j_{2}}^{+}}\right)-\left(y_{j_{2}}^{-}+\frac{1}{y_{j_{2}}^{-}}\right)=i \frac{\sqrt{2}}{g}, \quad j_{2}=1, \ldots, Q_{2} .
\end{aligned}
$$

By factorisability, the S-matrix for the scattering of the constituent magnons is simply a product of two-body factors. The formation of two boundstates of charges $Q_{1}$ and $Q_{2}$ corresponds to the pole in this multi-particle S-matrix appearing at,

$$
\begin{aligned}
& x_{j_{1}}^{-}=x_{j_{1}+1}^{+}, \quad j_{1}=1, \ldots, Q_{1}-1, \\
& y_{j_{2}}^{-}=y_{j_{2}+1}^{+}, \quad j_{2}=1, \ldots, Q_{2}-1 .
\end{aligned}
$$

The resulting boundstate spectral parameters $X^{ \pm}$and $Y^{ \pm}$can then be identified as:

$$
\begin{aligned}
& X^{+}=x_{1}^{+}, \quad X^{-}=x_{Q_{1}}^{-}, \\
& Y^{+}=y_{1}^{+}, \quad Y^{-}=y_{Q_{2}}^{-},
\end{aligned}
$$

where it is easy to check that the appropriate constraint equation for $X^{ \pm}$(i.e. Eqn (4) with $\left.Q=Q_{1}\right)$ is obeyed by virtue of (19) and (21) and similarly for $Y^{ \pm}$. Consistency of scattering in such that an integrable theory provides a simple recipe for extracting the boundstate S-matrix: it is simply the residue of the multi-particle scattering matrix of the constituent magnons at the pole specified above. This prescription is most familar in the context of relativistic field theories in $(1+1)$-dimensions [16, 17, but has also been applied successfully in the context of integrable spin chains [18]. In terms of the single magnon S-matrix $s\left(x^{+}, x^{-} ; y^{+}, y^{-}\right)$given in (9), the boundstate S-matrix is,

$$
S\left(Q_{1}, Q_{2}, p_{1}, p_{2}\right)=S\left(X^{+}, X^{-}, Y^{+}, Y^{-}\right)=\prod_{j_{1}=1}^{Q_{1}} \prod_{j_{2}=1}^{Q_{2}} s\left(x_{j_{1}}^{+}, x_{j_{2}}^{-} ; y_{j_{2}}^{+}, y_{j_{2}}^{-}\right) .
$$

It will be convenient to write $S$ as the product of two factors,

$$
S\left(Q_{1}, Q_{2}, p_{1}, p_{2}\right)=\hat{S} \times \Sigma .
$$

Here $\hat{S}$ is the contribution coming from the BDS factor $\hat{s}$ in the single magnon S-matrix, which is defined in (10). The remaining piece $\Sigma$ originates from the dressing factor $\sigma$ in the single magnon S-matrix, as defined in (12). We will consider these two factors in turn. 
The BDS piece of the boundstate S-matrix is straightforwardly obtained by direct evaluation of the product (25). The corresponding calculation for the XXX Heisenberg spin chain is reviewed in [18. The pole conditions (2122) lead to numerous cancellations between the $Q_{1} Q_{2}$ factors in the product. The remaining factors can be conveniently presented as,

$$
\hat{S}\left(Q_{1}, Q_{2}, p_{1}, p_{2}\right)=G\left(Q_{1}-Q_{2}\right)\left[\prod_{l=1}^{Q_{2}-1} G\left(Q_{1}-Q_{2}+2 l\right)\right]^{2} G\left(Q_{1}+Q_{2}\right),
$$

where

$$
G(Q)=\frac{\Delta U+\frac{i Q}{g \sqrt{2}}}{\Delta U-\frac{i Q}{g \sqrt{2}}} \quad \text { with } \quad \Delta U=U\left(p_{1}, Q_{1}\right)-U\left(p_{2}, Q_{2}\right)
$$

The singularities of the final answer (27) have a natural interpretation in terms of on-shell intermediate states. First, the simple pole of the factor $G\left(Q_{1}+Q_{2}\right)$ corresponds to the formation of a boundstate with $Q=Q_{1}+Q_{2}$ in the s-channel. This is a direct generalisation of the $Q=2$ pole in the S-matrix of two elementary magnons mentioned above. Similarly, the other simple pole in $\hat{S}$, which comes from the factor $G\left(Q_{1}-Q_{2}\right)$, precisely corresponds to the exchange of a boundstate with $Q=Q_{1}-Q_{2}>0$ in the t-channel. The set of $Q_{2}-1$ double poles in the boundstate S-matrix also have a standard explanation in $(1+1)$-dimensional scattering theory [28]: they correspond to anomalous thresholds. Specifically, the positions of the double poles are consistent with the kinematics of an intermediate state consisting of two on-shell boundstates with $Q=Q_{1}+l$ and $Q=Q_{2}-l$ respectively for $l=1,2, \ldots, Q_{2}-1$.

The second contribution to the boundstate scattering matrix, denoted $\Sigma$ in (26) comes from the dressing factors of the elementary magnon S-matrices appearing in the product (25). Here we find an even more complete cancellation of factors appearing in the product. In fact the final answer is simply that $\Sigma$ is identical as function of the higher conserved charges to the fundamental magnon dressing factor $\sigma$. Thus we have,

$$
\begin{gathered}
\Sigma(X, Y)=\exp [i \theta(X, Y)] \\
\theta(X, Y)=\sqrt{2} g \sum_{r=2}^{\infty} \sum_{n=0}^{\infty} c_{r, r+1+2 n}(g)\left[q_{r}(X) q_{r+1+2 n}(Y)-q_{r}(Y) q_{r+1+2 n}(X)\right],
\end{gathered}
$$

where the coefficient $c_{r, s}$ are the same as those appearing in (12). Thus the factor $\Sigma$ appearing in the boundstate S-matrix is equal to a universal function of the higher conserved charges. Equivalently we have

$$
\theta(X, Y)=\theta\left(X^{+}, X^{-} ; Y^{+}, Y^{-}\right)=\sqrt{2} g\left\{k\left(X^{+}, Y^{+}\right)+k\left(X^{-}, Y^{-}\right)-k\left(X^{+}, Y^{-}\right)-k\left(X^{-}, Y^{+}\right)\right\}
$$


where $k(X, Y)$ is the same function appearing in (14). As in the case of the single magnon S-matrix our knowledge of this function (or, equivalently of the coefficients $c_{r, s}$ ) is limited to the first two orders in the strong coupling expansion. As mentioned above, the general form (12) for the dressing factor originally arose as the most general integrable long-range deformation of the Heisenberg spin chain. In the present context it is interesting to note that it is essentially equivalent to the condition that the dressing factor should be the same universal function of the conserved charges for all BPS states in the theory. Indeed one could start by imposing this universality as a requirement and, after also taking account of unitarity and parity invariance, one would immediately be lead to the general form (12).

So far we have been considering the exact analytic expressions for the boundstate S-matrix. To compare our results with those of semiclassical string theory we need to take the strong coupling limit $g \rightarrow \infty$. As discussed in [19], the natural limit to take is one where the charges $Q_{1}$ and $Q_{2}$ also scale linearly with $g$. As a consequence, both terms under the square root in the dispersion relation (11) scale like $g^{2}$ and thus the energy $E$ has the appropriate coupling dependence for a semiclassical string state. Conveniently, the spectral parameters $X^{ \pm}$and $Y^{ \pm}$for boundstates with $Q=Q_{1}$ and $Q=Q_{2}$ respectively remain fixed in this limit. Our next goal is to calculate the leading asymptotics of the boundstate S-matrix as a function of the spectral parameters. As above we consider the two factors $\hat{S}$ and $\Sigma$ appearing in (26) in turn.

To take the strong coupling limit of $\hat{S}$, we begin by exponentiating the product appearing in (27) to obtain a sum in the exponent. As $g \rightarrow \infty$ this sum goes over to an integral, with the integration limits depending only on the sum and the difference between the charges $Q_{1}$ and $Q_{2}$. Interestingly, the leading contribution to $\hat{S}$ has the same general form (31) as that of the dressing factor. In particular, the final result can be given as,

$$
\hat{S}(X, Y) \sim \exp (i \hat{\theta}(X, Y))
$$

where

$$
\hat{\theta}\left(X^{+}, X^{-}, Y^{+}, Y^{-}\right)=\sqrt{2} g\left[\hat{k}\left(X^{+}, Y^{+}\right)+\hat{k}\left(X^{-}, Y^{-}\right)-\hat{k}\left(X^{+}, Y^{-}\right)-\hat{k}\left(X^{-}, Y^{+}\right)\right] .
$$

Here the function $\hat{k}$ is given by,

$$
\hat{k}(X, Y)=\left[\left(X+\frac{1}{X}\right)-\left(Y+\frac{1}{Y}\right)\right] \log \left[(X-Y)\left(1-\frac{1}{X Y}\right)\right] .
$$


The strong-coupling limit of the dressing factor $\Sigma$ is simply given by replacing the function $k(X, Y)$ appearing in (31) by the function $k_{0}(X, Y)$ given in (16). Collecting the results for the two factors we find the final result for the strong coupling limit of the boundstate S-matrix can be given as,

$$
S(X, Y) \sim \exp (i \Theta(X, Y))
$$

where

$$
\Theta\left(X^{+}, X^{-}, Y^{+}, Y^{-}\right)=\sqrt{2} g\left[K\left(X^{+}, Y^{+}\right)+K\left(X^{-}, Y^{-}\right)-K\left(X^{+}, Y^{-}\right)-K\left(X^{-}, Y^{+}\right)\right] .
$$

Here the function $K(X, Y)$ is given by,

$$
K(X, Y)=\hat{k}(X, Y)+k_{0}(X, Y)=\left[\left(X+\frac{1}{X}\right)-\left(Y+\frac{1}{Y}\right)\right] \log (X-Y) .
$$

In our previous paper [19] we showed that the magnon boundstates described above appear in string theory on $A d S_{5} \times S^{5}$ as classical solitons of the worldsheet action. For states in the $S U(2)$ sector we may restrict our attention to strings moving on an $\mathbb{R} \times S^{3}$ subspace of $A d S_{5} \times S^{5}$. The corresponding equations of motion together with the Virasoro constraint can be mapped onto the complex sine-Gordon ( $\mathrm{CsG}$ ) equation. Under this equivalence, the classical string solution corresponding to a magnon boundstate of charge $Q$ and momentum $p$ is mapped to a certain onesoliton solution of the CsG equation. The soliton in question has two parameters: a rapidity ${ }^{3} \theta$ and an additional rotation parameter $\alpha$. The dictionary between these parameters and the conserved quantities $E, Q$ and $p$ is,

$$
\begin{aligned}
& E=2 \sqrt{2} g \frac{\cos (\alpha) \cosh (\theta)}{\cos ^{2}(\alpha)+\sinh ^{2}(\theta)} \\
& Q=2 \sqrt{2} g \frac{\cos (\alpha) \sin (\alpha)}{\cos ^{2}(\alpha)+\sinh ^{2}(\theta)}
\end{aligned}
$$

and

$$
\cot \left(\frac{p}{2}\right)=\frac{\sinh (\theta)}{\cos (\alpha)}
$$

The CsG equation is completely integrable and has multi-soliton scattering solution which can be constructed explicitly via inverse scattering [29] or by the Hirota method [30]. The only effect of scattering is to induce a time delay for each soliton relative to free motion. For two solitons with rapidities $\theta_{1}$ and $\theta_{2}$ and rotation parameters $\alpha_{1}$ and $\alpha_{2}$ the COM frame is defined by the condition, $\cos \left(\alpha_{1}\right) \sinh \left(\theta_{1}\right)=-\cos \left(\alpha_{2}\right) \sinh \left(\theta_{2}\right)$. In this Lorentz frame the two solitons experience an equal time delay $\Delta T_{1}=\Delta T_{2}=\Delta T_{\mathrm{COM}}$ with [3],

$$
\Delta T_{\mathrm{COM}}=\frac{1}{\cos \left(\alpha_{1}\right) \sinh \left(\theta_{1}\right)} \log F(\Delta \theta, \Delta \alpha, \bar{\alpha}),
$$

\footnotetext{
${ }^{3}$ Not to be confused with the magnon rapidity $U(X)$ introduced above.
} 
where we define $\Delta \theta=\left(\theta_{1}-\theta_{2}\right) / 2, \Delta \alpha=\left(\alpha_{1}-\alpha_{2}\right) / 2$ and $\bar{\alpha}=\left(\alpha_{1}+\alpha_{2}\right) / 2$ and the function $F$ is given by,

$$
F(\Delta \theta, \Delta \alpha, \bar{\alpha})=\frac{\sinh (\Delta \theta+i \Delta \alpha) \sinh (\Delta \theta-i \Delta \alpha)}{\cosh (\Delta \theta+i \bar{\alpha}) \cosh (\Delta \theta-i \bar{\alpha})} .
$$

Time delays due to multiple soliton scattering are simply given by the sum of the delays experienced in each two-body collision. This is a consequence of integrability, and is a classical analog of the factorisability of the S-matrix. Indeed, the time delays determine the semiclassical approximation to the worldsheet S-matrix $S_{\text {string }}=\exp \left(i \Theta_{\text {string }}\right)$. In particular, if we express the S-matrix as a function of the energies $E_{1}$ and $E_{2}$ of the two excitations and their charges $Q_{1}$ and $Q_{2}$ we have 32 ,

$$
\Delta T_{1}=\frac{\partial \Theta_{\text {string }}}{\partial E_{1}}, \quad \Delta T_{2}=\frac{\partial \Theta_{\text {string }}}{\partial E_{2}} .
$$

Our aim here is to compare $S_{\text {string }}$ with the semiclassical limit of the magnon boundstate Smatrix computed above. Equivalently we can use the boundstate S-matrix to compute the time delay in boundstate scattering directly and compare with the COM frame expression for $\Delta T_{1}$ and $\Delta T_{2}$ presented in (41) above. To do so, one has to first express $\Theta\left(X^{+}, X^{-}, Y^{+}, Y^{-}\right)$in terms of the charges $Q_{1}, Q_{2}$ and the energies $E_{1}, E_{2}$ using the relations:

$$
\begin{aligned}
& E_{1}=\frac{g}{i \sqrt{2}}\left[\left(X^{+}-\frac{1}{X^{+}}\right)-\left(X^{-}-\frac{1}{X^{-}}\right)\right], \\
& E_{2}=\frac{g}{i \sqrt{2}}\left[\left(Y^{+}-\frac{1}{Y^{+}}\right)-\left(Y^{-}-\frac{1}{Y^{-}}\right)\right], \\
& Q_{1}=\frac{g}{i \sqrt{2}}\left[\left(X^{+}+\frac{1}{X^{+}}\right)-\left(X^{-}+\frac{1}{X^{-}}\right)\right], \\
& Q_{2}=\frac{g}{i \sqrt{2}}\left[\left(Y^{+}+\frac{1}{Y^{+}}\right)-\left(Y^{-}+\frac{1}{Y^{-}}\right)\right] .
\end{aligned}
$$

We then define,

$$
\Delta \tau_{1}=\frac{\partial \Theta}{\partial E_{1}}, \quad \Delta \tau_{2}=\frac{\partial \Theta}{\partial E_{2}},
$$

while keeping the charges $Q_{1}$ and $Q_{2}$ fixed. Here we present the results of explicit differentiations exclusively in terms of spectral parameters:

$$
\begin{aligned}
& \Delta \tau_{1}=i \frac{\left(\left(X^{+}\right)^{2}-1\right)\left(\left(X^{-}\right)^{2}-1\right)}{\left(\left(X^{+}\right)^{2}-\left(X^{-}\right)^{2}\right)} \log \left(\frac{X^{+}-Y^{+}}{X^{+}-Y^{-}} \frac{X^{-}-Y^{-}}{X^{-}-Y^{+}}\right)-\left(\frac{1}{Y^{+}}-\frac{1}{Y^{-}}\right) \frac{\left(X^{+} X^{-}+1\right)}{\left(X^{+}+X^{-}\right)}, \\
& \Delta \tau_{2}=-i \frac{\left(\left(Y^{+}\right)^{2}-1\right)\left(\left(Y^{-}\right)^{2}-1\right)}{\left(\left(Y^{+}\right)^{2}-\left(Y^{-}\right)^{2}\right)} \log \left(\frac{X^{+}-Y^{+}}{X^{+}-Y^{-}} \frac{X^{-}-Y^{-}}{X^{-}-Y^{+}}\right)+\left(\frac{1}{X^{+}}-\frac{1}{X^{-}}\right) \frac{\left(Y^{+} Y^{-}+1\right)}{\left(Y^{+}+Y^{-}\right)} .
\end{aligned}
$$


All that remains is to compare with the CsG time delays. Combining the identities (44)-(47) and the relations (38) and (39), one can express the spectral parameters of the magnon boundstates in terms of ${ }^{4} \theta_{i}$ and $\alpha_{i}$,

$$
\begin{aligned}
& X^{ \pm}=\operatorname{coth}\left(\frac{\theta_{1}}{2} \pm i\left(\frac{\alpha_{1}}{2}-\frac{\pi}{4}\right)\right), \\
& Y^{ \pm}=\operatorname{coth}\left(\frac{\theta_{2}}{2} \pm i\left(\frac{\alpha_{2}}{2}-\frac{\pi}{4}\right)\right) .
\end{aligned}
$$

These expressions in turn yield

$$
F(\Delta \theta, \Delta \alpha, \bar{\alpha}) \equiv F\left(X^{+}, X^{-}, Y^{+}, Y^{-}\right)=\frac{\left(X^{+}-Y^{+}\right)\left(X^{-}-Y^{-}\right)}{\left(X^{+}-Y^{-}\right)\left(X^{-}-Y^{+}\right)} .
$$

Now comparing (41) with (49) and (50), taking into account the COM frame condition, we can see that the time-delays for boundstate scattering agree with those of CsG solitons up to a specific non-logarithmic term,

$$
\Delta \tau_{1}=\Delta T_{1}+\left(\frac{1}{Y^{+}}-\frac{1}{Y^{-}}\right) \frac{\left(X^{+} X^{-}+1\right)}{\left(X^{+}+X^{-}\right)} .
$$

Upon integration with respect to $E_{1}$, we can obtain the relation between the scattering phases:

$$
\Theta_{\text {string }}=\Theta\left(X^{+}, X^{-}, Y^{+}, Y^{-}\right)+\left(E_{2}-Q_{2}\right) p_{1}
$$

The non-logarithmic term in (49) integrates up to give the difference term above that is a direct generalisation of the one in eqn. (3.33) of [9]. As in that case, the difference can be accounted for by taking into account the different effective length of the excitation on the both sides of the correspondence and is irrelevant for the Bethe ansatz equations. One can also check that the expressions in (49) and (50) correctly satisfy $\Delta \tau_{1}=\Delta \tau_{2}$ in the COM frame.

The authors acknowledge useful discussions with Gleb Arutyunov, Diego Hofman, Juan Maldacena and Radu Roiban. We are grateful to Radu Roiban for providing us with a preliminary version of 33. The authors would also like to thank Rui Fernando Lima Matos for collaboration on some of the work presented here. HYC is supported by a Benefactors' scholarship from St. John's College, Cambridge. ND is supported by a PPARC Senior Fellowship. HYC would like to thank National Taiwan University, Physics Department for the hospitality, where part of the work was completed. KO is very grateful to University of Cambridge, Centre for Mathematical Sciences, where part of the work was done, for its warm hospitality.

\footnotetext{
${ }^{4}$ In obtaining these expression one needs to solve quadratic equations. The appropriate root of the quadratic is selected by demanding that the corresponding state has positive energy.
} 


\section{References}

[1] J. A. Minahan and K. Zarembo, "The Bethe-ansatz for N = 4 super Yang-Mills," JHEP 0303 (2003) 013 arXiv:hep-th/0212208.

[2] N. Beisert and M. Staudacher, "The N = 4 SYM integrable super spin chain," Nucl. Phys. B 670 (2003) 439 arXiv:hep-th/0307042.

N. Beisert, C. Kristjansen and M. Staudacher, "The dilatation operator of N = 4 super YangMills theory," Nucl. Phys. B 664 (2003) 131 arXiv:hep-th/0303060.

[3] G. Mandal, N. V. Suryanarayana and S. R. Wadia, "Aspects of semiclassical strings in AdS(5)," Phys. Lett. B 543, 81 (2002) arXiv:hep-th/0206103.

I. Bena, J. Polchinski and R. Roiban, "Hidden symmetries of the AdS(5) x S**5 superstring," Phys. Rev. D 69, 046002 (2004) arXiv:hep-th/0305116.

[4] N. Beisert, V. Dippel and M. Staudacher, "A novel long range spin chain and planar N $=4$ super Yang-Mills," JHEP 0407 (2004) 075 arXiv:hep-th/0405001.

[5] G. Arutyunov, S. Frolov and M. Staudacher, "Bethe ansatz for quantum strings," JHEP 0410 (2004) 016 arXiv:hep-th/0406256.

[6] M. Staudacher, "The factorized S-matrix of CFT/AdS," JHEP 0505 (2005) 054 arXiv:hep-th/0412188.

[7] N. Beisert and M. Staudacher, "Long-range $\operatorname{PSU}(2,2 \mid 4)$ Bethe ansaetze for gauge theory and strings," Nucl. Phys. B 727 (2005) 1 arXiv:hep-th/0504190.

[8] N. Beisert, "The $\mathfrak{s u}(2 \mid 2)$ dynamic S-matrix," arXiv:hep-th/0511082,

[9] D. M. Hofman and J. M. Maldacena, "Giant magnons," arXiv:hep-th/0604135

[10] N. Mann and J. Polchinski, "Bethe ansatz for a quantum supercoset sigma model," Phys. Rev. D 72 (2005) 086002 arXiv:hep-th/0508232.

J. Ambjorn, R. A. Janik and C. Kristjansen, "Wrapping interactions and a new source of corrections to the spin-chain / string duality," Nucl. Phys. B 736, 288 (2006) arXiv:hep-th/0510171.

T. Klose and K. Zarembo, "Bethe ansatz in stringy sigma models," arXiv:hep-th/0603039.

N. Gromov, V. Kazakov, K. Sakai and P. Vieira, "Strings as multi-particle states of quantum sigma-models," arXiv:hep-th/0603043 
G. Arutyunov and A. A. Tseytlin, "On highest-energy state in the su(1|1) sector of $\mathrm{N}=4$ super Yang-Mills arXiv:hep-th/0603113.

[11] R. A. Janik, "The AdS(5) x $\mathrm{S}^{* *} 5$ superstring worldsheet S-matrix and crossing symmetry," Phys. Rev. D 73 (2006) 086006 arXiv:hep-th/0603038.

[12] G. Arutyunov and S. Frolov, "On AdS(5) x S**5 string S-matrix," arXiv:hep-th/0604043.

[13] N. Dorey, "Magnon bound states and the AdS/CFT correspondence," arXiv:hep-th/0604175.

[14] A. Santambrogio and D. Zanon, "Exact anomalous dimensions of $\mathrm{N}=4$ Yang-Mills operators with large R charge," Phys. Lett. B 545 (2002) 425 arXiv:hep-th/0206079.

D. Berenstein, D. H. Correa and S. E. Vazquez, "All loop BMN state energies from matrices," JHEP 0602 (2006) 048 arXiv:hep-th/0509015.

[15] N. Beisert and T. Klose, "Long-range gl(n) integrable spin chains and plane-wave matrix theory," J. Stat. Mech. 0607 (2006) P006 arXiv:hep-th/0510124.

[16] A. B. Zamolodchikov and A. B. Zamolodchikov, "Factorized S-Matrices In Two Dimensions As The Exact Solutions Of Certain Relativistic Quantum Field Models," Annals Phys. 120 (1979) 253.

[17] M. Karowski, "On The Bound State Problem In (1+1)-Dimensional Field Theories," Nucl. Phys. B 153 (1979) 244.

[18] L. D. Faddeev, "How Algebraic Bethe Ansatz works for integrable model," arXiv:hep-th/9605187,

[19] H. Y. Chen, N. Dorey and K. Okamura, "Dyonic giant magnons," arXiv:hep-th/0605155.

[20] G. Arutyunov, S. Frolov and M. Zamaklar, "Finite-size effects from giant magnons," arXiv:hep-th/0606126.

[21] J. A. Minahan, A. Tirziu and A. A. Tseytlin, "Infinite spin limit of semiclassical string states," arXiv:hep-th/0606145.

[22] M. Spradlin and A. Volovich, "Dressing the giant magnon," arXiv:hep-th/0607009.

[23] N. P. Bobev and R. C. Rashkov, "Multispin giant magnons," arXiv:hep-th/0607018.

[24] M. Kruczenski, J. Russo and A. A. Tseytlin, "Spiky strings and giant magnons on S5," arXiv:hep-th/0607044. 
[25] G. Arutyunov and M. Staudacher, "Matching higher conserved charges for strings and spins," JHEP 0403 (2004) 004 arXiv:hep-th/0310182.

[26] J. A. Minahan, Talk at 11th Marcel Grossman Meeting, 2006.

[27] R. Hernandez and E. Lopez, "Quantum corrections to the string Bethe ansatz," arXiv:hep-th/0603204.

[28] S. R. Coleman and H. J. Thun, "On The Prosaic Origin Of The Double Poles In The SineGordon S Matrix," Commun. Math. Phys. 61, 31 (1978).

[29] H. J. de Vega and J. M. Maillet, "Semiclassical Quantization Of The Complex Sine-Gordon Field Theory," Phys. Rev. D 28 (1983) 1441.

[30] B. S. Getmanov, "Integrable Model For The Nonlinear Complex Scalar Field With The Nontrivial Asymptotics Of N - Soliton Solutions," Theor. Math. Phys. 38 (1979) 124 [Teor. Mat. Fiz. 38 (1979) 186].

B. S. Getmanov, "Integrable Two-Dimensional Lorentz Invariant Nonlinear Model Of Complex Scalar Field (Complex Sine-Gordon Ii)," Theor. Math. Phys. 48 (1982) 572 [Teor. Mat. Fiz. 48 (1981) 13].

[31] N. Dorey and T. J. Hollowood, "Quantum scattering of charged solitons in the complex sineGordon model," Nucl. Phys. B 440 (1995) 215 arXiv:hep-th/9410140.

[32] R. Jackiw and G. Woo, "Semiclassical Scattering Of Quantized Nonlinear Waves," Phys. Rev. D 12 (1975) 1643.

[33] R. Roiban, "Dyonic giant magnon scattering in gauge theory and string theory" to appear. 